\title{
A new model relating secondary nucleation rate and supersaturation
}

\author{
Clifford Y. Tai ${ }^{*}$, Cheng-Yi Shih \\ Department of Chemical Engineering, National Taiwan University, Taipei 10764, Taiwan, ROC
}

Received 25 October 1993; accepted 5 October 1995

\begin{abstract}
A new model of secondary nucleation rate regarding the effects of supersaturation is presented. The model takes account of the interfacial supersaturation, which influences the number of nuclei that is produced, and the overall supersaturation, which determines the fraction of nuclei that survived. The interfacial supersaturation can be replaced by the crystal growth rate so that the nucleation rate equation includes the overall supersaturation and crystal growth rate. This is different from the nucleation models used in the literature with only one of them appearing. Simplified models of the nucleation rate are derived and presented.
\end{abstract}

Secondary nucleation has been recognized as the dominant mechanism of nucleation in a suspension crystallizer. This was, however, uncertain till the early work on contact nucleation, or collision breeding, was reported. Using different experimental techniques in two separate laboratories, Strickland-Constable and co-workers [1-3] and McCabe and coworkers [4-7] presented evidences showing that contact nucleation was dominating at low supersaturation. They also demonstrated the dual role of supersaturation in the determination of the secondary nucleation rate; both on the generation and development stages of nuclei. The supersaturation is referred to the overall supersaturation, $\mathrm{C}-\mathrm{C}^{*}$. However, there is a hypothesis brought up by Tai et al. [8] that the number of nuclei which survive in the development period is determined by the overall supersaturation but the number of nuclei generated by the contact is influenced by the interfacial supersaturation. The interfacial supersaturation is a variable

\footnotetext{
"Corresponding author.
}

appearing in the two-step model of crystal growth, which can be expressed mathematically as:

$G=k_{\mathrm{d}}\left(\sigma-\sigma_{\mathrm{i}}\right)$, diffusion,

$G=k_{\mathrm{r}}\left(\sigma_{\mathrm{i}}\right)^{n_{\mathrm{g}}}, \quad$ surface reaction,

where $\sigma$, i.e., $\left(C-C^{*}\right) / C^{*}$, is the dimensionless overall supersaturation, and $\sigma_{\mathrm{i}}$, i.e., $\left(C_{\mathrm{i}}-C^{*}\right) / C^{*}$, the dimensionless interfacial supersaturation. Since the driving force $\sigma-\sigma_{\mathrm{i}}$ is for the diffusion, it is no longer effective in generating secondary nuclei which come from the adsorption layer. Thus, the number of nuclei generated by the contact mechanism should be a function of interfacial supersaturation instead of overall supersaturation. It should be noted that the interfacial supersaturation is related to the overall supersaturation in different ways. The interfacial supersaturation will change with the overall supersaturation in the same direction, or inthe opposite direction as demonstrated by Tai et al. [8]. The aim of this communication is to show how the interfacial supersaturation would affect the rate of secondary nucleation. 
Ottens and de Jong [9] have developed a model of contact nucleation in a stirred-tank crystallizer. They began with the expression for the nucleation rate of $n \mathrm{~d} L$ crystals between size $L$ and $L+\mathrm{d} L$.

$\mathrm{d} B^{0}=k_{3}\left(C-C^{*}\right)^{q} \omega_{L} E_{L} n \mathrm{~d} L$,

where $\omega_{\mathrm{L}}$ is the crystal-impeller contact frequency and $E_{L}$ the impact energy associated with the contact. They assumed that both the number of nuclei generated and the portion of nuclei survived are functions of the overall supersaturation. According to the hypothesis proposed by Tai et al. [8], however, the number of crystals produced per unit time in the generation stage is

$\mathrm{d} J=k_{4} \sigma_{\mathrm{i}}^{n_{\mathrm{b}}} \omega_{L} E_{L} n \mathrm{~d} L$.

Eq. (4) is similar to Eq. (3), except for $C-C^{*}$ being replaced by $\sigma_{\mathrm{i}}$.

For all sizes of crystals in suspension, the total number of nuclei produced is

$J=\int_{L_{x}}^{\infty} k_{4} \sigma_{\mathrm{i}}{ }^{{ }^{n}} \omega_{L} E_{L} n \mathrm{~d} L$,

where $L_{x}$ is the minimum crystal size which contributes to secondary nucleation.
Bauer et al. [10] have assumed that the crystalimpeller contact frequency for a homogeneous suspension is a function of the magma circulation rate and target efficiency:

$\omega_{L}=k_{\mathrm{w}}\left(\phi_{\mathrm{v}} / V_{\mathrm{c}}\right) \eta_{\mathrm{t}}=k_{\mathrm{w}}\left(k_{\mathrm{p}} n_{\mathrm{r}} D_{\mathrm{r}}^{3} / V_{\mathrm{c}}\right) \eta_{\mathrm{t}}$,

where $\phi_{\mathrm{v}}$ is the pumping capacity of the impeller, $V_{\mathrm{c}}$ the total volume of the crystallizer, $\eta_{t}$ the target efficiency, $n_{\mathrm{t}}$ the impeller speed, and $D_{\mathrm{t}}$ the impeller diameter.

The impact energy $E_{\mathrm{L}}$ is proportional to the crystal mass of size $L$ and the square of the tip speed of the impeller $V_{\mathrm{t}}[11]$ :

$E_{L} \propto m_{L}\left(V_{\mathrm{t}}\right)^{2} \propto m_{L}\left(n_{\mathrm{r}} D_{\mathrm{r}}\right)^{2}$,

and since

$m_{L}=k_{\mathrm{v}} \rho_{\mathrm{s}} L^{3}$

Eq. (5) may be written as

$J=k_{5} \int_{L_{x}}^{\infty}\left(n_{\mathrm{r}}^{3} D_{\mathrm{r}}^{5} / V_{\mathrm{c}}\right) \sigma_{\mathrm{i}}{ }^{n_{\mathrm{b}}} \eta_{\mathrm{t}} L^{3} n \mathrm{~d} L$.

For a given crystallizer operating at constant agitation rate, Eq. (9) becomes

$J=k_{6} \int_{L_{x}}^{\infty} \sigma_{\mathrm{i}}^{n_{\mathrm{b}}} \eta_{\mathrm{t}} L^{3} n \mathrm{~d} L$,

Table 1

Expression of reduced secondary nucleation rate

The general secondary nucleation rate equation

$B^{0}=k_{7} \sigma^{s} \int_{L_{x}}^{\infty} \sigma_{i}^{n_{\mathrm{h}}} L^{3} n \mathrm{~d} L$

Limiting condition

Reduced nucleation rate

Crystal growth process is controlled

Reduced nucleation rate

by surface integration, $\sigma=\sigma_{i}$.

$k_{8} M_{\mathrm{T}} \sigma^{\prime}$ where $r=s+n_{\mathrm{b}}$

(12)

Crystal growth process is controlled

Nucleation rate is insignificant

by diffusion, $\sigma_{\mathrm{i}}=0$.

Surface integration rate is first order

$k_{9} M_{\mathrm{T}} \sigma^{r}$

with respect to interfacial supersaturation

and McCabe $\Delta$ law holds.

Surface integration rate is second order

with respect to interfacial supersaturation and McCabe $\Delta$ law holds.

Minimum size required for generating secondary nuclei and population density curve is nearly straight for crystals larger than the minimum size.

$$
k_{10} \sigma^{s}\left(-K_{\mathrm{T}}+\sqrt{K_{\mathrm{T}}^{2}+4 K_{\mathrm{T}} \sigma}\right)^{n} M_{\mathrm{T}}
$$$$
\text { where } K_{\mathrm{T}}=k_{\mathrm{d}} / k_{\mathrm{r}} \text {, is a constant. }
$$

$k_{11} \sigma^{s} \sigma_{\mathrm{i}}{ }^{n} M_{\mathrm{T}}$

$k_{12} \sigma^{s} G^{\beta} M_{\mathrm{T}}$ 
where $J$ is the production rate of particles in the generation stage. If the effect of supersaturation on the survival stage is considered and the target efficiency is neglected, i.e. $\eta_{\mathrm{t}}=1$, the nucleation rate can be expressed as:

$B^{0}=k_{7} \sigma^{s} \int_{L_{x}}^{\infty} \sigma_{\mathrm{i}}^{n_{\mathrm{b}}} L^{3} n \mathrm{~d} L$

In Eq. (11) the relation between the survived fraction of nuclei and the overall supersaturation is assumed to be a power-law function as proposed by Garside and Davey [12].

Direct integration of Eq. (11) is impossible because $n_{\mathrm{b}}$ is unknown for most systems and $\sigma_{\mathrm{i}}$ is a complex function of $L$ and $\sigma ; \sigma_{\mathrm{i}}$ varies with the particle size of the crystals under the constraint of constant $\sigma$. However, Eq. (11) can be reduced to a simple form at limiting conditions. The expressions of reduced secondary nucleation rate are summarized in Table 1.

To use Eq. (15), $\sigma_{i}$ should be determined. In the literature the mass-transfer coefficient can be obtained from the crystal dissolution [13] or it can be estimated by a modified Froessling equation [14]. Practically, Eq. (16) is much easier to use than Eq. (15) because crystal growth rate $G$ can be determined from the nearly straight portion of the population density curve.

So far, only a few experimental results in the literature can be used to support the hypothesis, but they are not conclusive. In order to gather more experimental evidences, experiments should be carefully designed. For instance, both the mass-transfer and surface-reaction resistance should be significant for the chosen system; or a suitable type of crystallizer other than a stirred tank should be used in order not to eliminate the mass-transfer resistance. Effort in this direction is continued in our laboratory.

\section{Nomenclature}

$J \quad$ number of crystals produced per unit time in the

generation stage

$k_{\mathrm{r}} \quad$ surface-integration rate constant

$k_{\mathrm{v}} \quad$ volume shape factor of the crystal

$L \quad$ crystal size

$M_{\mathrm{T}} \quad$ magma density

$m_{\mathrm{L}} \quad$ mass of a crystal of size $L$

$n \quad$ crystal population density

$n_{\mathrm{r}} \quad$ agitation rate

$s \quad$ the exponent of overall supersaturation in Eq. (11)

$V_{c} \quad$ crystallizer volume

$V_{\mathrm{t}} \quad$ tip speed of impeller

$\beta \quad n_{\mathrm{b}} / n_{\mathrm{g}}$

$\eta_{\mathrm{t}} \quad$ target efficiency

$\rho \quad$ solution density

$\rho_{\mathrm{s}} \quad$ crystal density

$\phi_{\mathrm{v}} \quad$ pumping capacity of the impeller [no. of crystal $/ \mathrm{s}$ ( $\mathrm{m}^{3}$ of suspension)]

$[\mathrm{m} / \mathrm{s}]$

[-]

[m]

[kg $/ \mathrm{m}^{3}$ of suspension]

$[\mathrm{kg}]$

[no. of crystal $/ \mathrm{m}^{3} \cdot \mu \mathrm{m}$ ]

[rpm]

[-]

$\left[\mathrm{m}^{3}\right]$

[m/s]

[-]

[-]

$\left[\mathrm{kg} / \mathrm{m}^{3}\right]$

$\left[\mathrm{kg} / \mathrm{m}^{3}\right]$

$\left[\mathrm{m}^{3} / \mathrm{s}\right]$ 


\section{References}

[1] D.P. Lal, R.E.A. Mason and R.F. Strickland-Constable, J. Crystal Growth 5 (1969) 1.

[2] H. Garabedian and R.F. Strickland-Constable, J. Crystal Growth 13/14 (1972) 506.

[3] H. Garabedian and R.F. Strickland-Constable, J. Crystal Growth 12 (1972) 53.

[4] N.A. Clontz and W.L. McCabe, Chem. Eng. Progr. Symp. Ser. 67 (1971) 6.

[5] R.T. Johnson, R.W. Rousseau and W.L. McCabe, AIChE Symp. Ser. 68 (1972) 31.

[6] C.Y. Tai, W.L. McCabe and R.W. Rousseau, AIChE J. 21 (1975) 351.

[7] R.W. Rousseau, W.L. McCabe and C.Y. Tai, AIChE J. 21 (1975) 1071.
[8] C.Y. Tai, J.F. Wu and R.W. Rousseau, J. Crystal Growth 116 (1992) 294.

[9] E.P.K. Ottens and E.J. de Jong, Ind. Eng. Chem. Fundam. 12 (1973) 179.

[10] L.G. Bauer, R.W. Rousseau and W.L. McCabe, AIChE J. 20 (1974) 653.

[11] R.C. Bennett, H. Fiedelman and A.D. Randolph, Chem. Eng. Progr. 69 (1973) 86.

[12] J. Garside and R.J. Davey, Chem. Eng. Commun. 4 (1980) 393.

[13] J. Garside, J.W. Mullin and S.N. Das, Ind. Eng. Chem. Fundam. 13 (1974) 299.

[14] C.Y. Tai, C.Y. Chen and J.F. Wu, Chem. Eng. Commun. 56 (1987) 329. 